KYUNGPOOK Math. J. 54(2014), 103-111

http://dx.doi.org/10.5666/KMJ.2014.54.1.103

\title{
Approximation of Common Fixed Points of Mean Non- expansive Mapping in Banach Spaces
}

\author{
Gu ZhaOHui \\ Cisco School of Informatics, Guangdong University of Foreign Studies, Guangzhou, \\ 510420, P. R. China \\ e-mail : zhgugz@163.com \\ Li YONGJIN* \\ Department of Mathematics, Sun Yat-Sen University, Guangzhou, 510275 P. R. \\ China \\ e-mail : stslyj@mail.sysu.edu.cn
}

Abstract. Let $X$ be a uniformly convex Banach space, and $S, T$ be pair of mean nonexpansive mappings. Some necessary and sufficient conditions are given for Ishikawa iterative sequence converge to common fixed points, and we prove that the sequence of Ishikawa iterations associated with $S$ and $T$ converges to the common fixed point of $S$ and $T$. This generalizes former results proved by Z. Gu and Y. Li [4].

\section{Introduction}

Let $X$ be a Banach space and $S, T$ be mappings from $X$ to $X$. In [8] the Ishikawa iteration sequence $\left\{x_{n}\right\}$ of $T$ was defined by

$$
\begin{gathered}
y_{n}=\left(1-\beta_{n}\right) x_{n}+\beta_{n} T x_{n} \\
x_{n+1}=\left(1-\alpha_{n}\right) x_{n}+\alpha_{n} T y_{n}
\end{gathered}
$$

Where $x_{0} \in X, \alpha_{n}, \beta_{n} \in[0,1]$.

The pair of mean non-expansive mappings was introduced by Bose in [2].

(1.3) $\|S x-T y\| \leq a\|x-y\|+b\{\|x-S x\|+\|y-T y\|\}+c\{\|x-T y\|+\|y-S x\|\}$

* Corresponding Author.

Received March 19, 2012; accepted November 7, 2012.

2010 Mathematics Subject Classification: 26D15.

Key words and phrases: Pair of mean non-expansive mappings, Ishikawa iteration, Common fixed point. 
for all $x, y \in X, a, b, c \in[0,1], a+2 b+2 c \leq 1$.

The Ishikawa iteration sequence $\left\{x_{n}\right\}$ of $S$ and $T$ was defined by

$$
\begin{gathered}
y_{n}=\left(1-\beta_{n}\right) x_{n}+\beta_{n} S x_{n} \\
x_{n+1}=\left(1-\alpha_{n}\right) x_{n}+\alpha_{n} T y_{n}
\end{gathered}
$$

Where $x_{0} \in X, \alpha_{n}, \beta_{n} \in[0,1]$.

The problems about common fixed point for pair of mappings as an important part of the fixed point theory have been studied by many authors, see [2-4,6-14], for more details. In [2], S. C. Bose defined the pair of mean non-expansive mappings, and proved the existence of the fixed point in Banach spaces. In particular, he proved the following theorem.

Theorem 1.1.[2] Let $X$ be a uniformly convex Banach space and $K$ a non-empty closed convex subset of $X, S: K \rightarrow K$ and $T: K \rightarrow K$ are pair of mean nonexpansive mappings, and $c \neq 0$, then

(i) $S$ and $T$ have a common fixed point $u$.

(ii) Further if $b \neq 0$, then

(a) $u$ is the unique common fixed point and unique as a fixed point of each $S$ and $T$.

(b) the sequence $\left\{x_{n}\right\}$ defined by $x_{1}=S x_{0}, x_{2}=T x_{1}, x_{3}=S x_{2} \cdots$ for any $x_{0} \in K$, converges strongly to $u$.

In [14], Z. Gu and Y. Li proved the following theorem.

Theorem 1.2. Let $X$ be a uniformly convex Banach space, $S: X \rightarrow X$ and $T: X \rightarrow X$ are pair of mean non-expansive with a nonempty common fixed points set, if $b>0,0<\alpha \leq \alpha_{n} \leq \frac{1}{2}, 0 \leq \beta_{n} \leq \beta<1$, then the Ishikawa sequence $\left\{x_{n}\right\}$ converges to the common fixed point of $S$ and $T$.

It is our purpose in this paper to give some necessary and sufficient conditions for Ishikawa iterative sequence converge to common fixed points, and consider the iterative scheme, which converges to a common fixed point of the pair of mean nonexpansive mappings. Our Theorem 2.1 extends and improves the corresponding results in [14].

To obtain the main results of the paper, we prove the following lemmas.

Lemma 1.3.[13] Let $X$ be a Banach space. Then $X$ is uniformly convex if and only if for any given number $\rho>0$, the square norm $\|\cdot\|^{2}$ of $X$ in uniformly convex on $B_{\rho}$, the closed unit ball at the origin with radius $\rho$; namely, there exist a continuous strictly increasing function $\varphi:[0, \infty) \rightarrow[0, \infty)$ with $\varphi(0)=0$ such that 
for all $x, \alpha x+(1-\alpha) y\left\|^{2} \leq \alpha\right\| x\left\|^{2}+(1-\alpha)\right\| y \|^{2}-\alpha(1-\alpha) \varphi(\|x-y\|)$,

Lemma 1.4.[14] Let $X$ be a Banach space, $S: X \rightarrow X$ and $T: X \rightarrow X$ are pair of mean non-expansive mappings with a common fixed point, then for any $x_{0} \in X$, the Ishikawa sequence $\left\{x_{n}\right\}$ associated with $S$ and $T$ is bounded.

Proof. For a common fixed point $p$ of $S$ and $T$, we have

$$
\begin{array}{ll} 
& \|T x-p\|=\|T x-S p\| \\
\leq \quad & a\|x-p\|+b\{\|x-T x\|+\|p-S p\|\} \\
& +c\{\|x-S p\|+\|p-T x\|\} \\
\leq & a\|x-p\|+b\{\|x-p\|+\|p-T x\|\} \\
& +c\{\|x-S p\|+\|p-T x\|\}
\end{array}
$$

Let $L=\frac{a+b+c}{1-b-c}$, by $a+2 b+2 c \leq 1$, it is easy to see that $a+b+c \leq 1-b-c$, thus $0 \leq L \leq 1$, and $\|T x-p\| \leq L\|x-p\| \leq\|x-p\|$.

Similarly, we have $\|S x-p\| \leq L\|x-p\| \leq\|x-p\|$.

$$
\begin{aligned}
\left\|x_{n+1}-p\right\| \leq & \left\|\left(1-\alpha_{n}\right) x_{n}+\alpha_{n} T y_{n}-p\right\| \\
= & \left\|\left(1-\alpha_{n}\right)\left(x_{n}-p\right)+\alpha_{n}\left(T y_{n}-p\right)\right\| \\
\leq & \left(1-\alpha_{n}\right)\left\|x_{n}-p\right\|+\alpha_{n}\left\|T y_{n}-p\right\| \\
\leq & \left(1-\alpha_{n}\right)\left\|x_{n}-p\right\|+\alpha_{n} L\left\|y_{n}-p\right\| \\
= & \left(1-\alpha_{n}\right)\left\|x_{n}-p\right\|+\alpha_{n}\left\|\left(1-\beta_{n}\right) x_{n}+\beta_{n} S x_{n}-p\right\| \\
= & \left(1-\alpha_{n}\right)\left\|x_{n}-p\right\|+\alpha_{n}\left\|\left(1-\beta_{n}\right)\left(x_{n}-p\right)+\beta_{n}\left(S x_{n}-p\right)\right\| \\
\leq & \left(1-\alpha_{n}\right)\left\|x_{n}-p\right\|+\alpha_{n}\left(1-\beta_{n}\right)\left\|x_{n}-p\right\| \\
& +\alpha_{n} \beta_{n}\left\|S x_{n}-p\right\| \\
\leq & \left(1-\alpha_{n}\right)\left\|x_{n}-p\right\|+\alpha_{n}\left(1-\beta_{n}\right)\left\|x_{n}-p\right\| \\
& +\alpha_{n} \beta_{n}\left\|x_{n}-p\right\| \\
= & \left(\left(1-\alpha_{n}\right)+\alpha_{n}\left(1-\beta_{n}\right)+\alpha_{n} \beta_{n}\right)\left\|x_{n}-p\right\| \\
= & \left\|x_{n}-p\right\|
\end{aligned}
$$

So

$$
\left\|x_{n+1}-p\right\| \leq\left\|x_{n}-p\right\| \leq\left\|x_{n-1}-p\right\| \leq \ldots \leq\left\|x_{0}-p\right\|
$$

Thus, $\left\{x_{n}\right\}$ is bounded.

\section{Section 2}

First, we give some necessary and sufficient conditions for Ishikawa iterative 
sequence converge to common fixed points.

Theorem 2.1. Let $X$ be a Banach space, $S: X \rightarrow X$ and $T: X \rightarrow X$ are pair of mean non-expansive with a nonempty common fixed points set, if $b>0, c>0$, then the necessary and sufficient conditions that the Ishikawa sequence $\left\{x_{n}\right\}$ converges to the common fixed point of $S$ and $T$ is:

$$
\lim _{n \rightarrow \infty} \inf \left\|x_{n}-T y_{n}\right\|=0 .
$$

Proof. For the first step, we will prove the sufficiency.

Let us first prove $\lim _{n \rightarrow \infty}\left\|x_{n}-S x_{n}\right\|=0$. Since

$$
\begin{aligned}
\left\|x_{n}-S x_{n}\right\| \leq & \left\|x_{n}-T y_{n}\right\|+\left\|T y_{n}-S x_{n}\right\| \\
\leq & \left\|x_{n}-T y_{n}\right\|+a\left\|x_{n}-y_{n}\right\|+b\left\{\left\|x_{n}-S x_{n}\right\|+\left\|y_{n}-T y_{n}\right\|\right\} \\
& +c\left\{\left\|x_{n}-T y_{n}\right\|+\left\|y_{n}-S x_{n}\right\|\right\} \\
= & (1+c)\left\|x_{n}-T y_{n}\right\|+a\left\|x_{n}-y_{n}\right\|+b\left\|x_{n}-S x_{n}\right\| \\
& +b\left\|y_{n}-T y_{n}\right\|+c\left\|y_{n}-S x_{n}\right\| \\
= & (1+c)\left\|x_{n}-T y_{n}\right\|+a\left\|\left(1-\beta_{n}\right) x_{n}+\beta_{n} S x_{n}-x_{n}\right\| \\
& +b\left\|x_{n}-S x_{n}\right\|+b\left\|\left(1-\beta_{n}\right) x_{n}+\beta_{n} S x_{n}-T y_{n}\right\| \\
& +c\left\|\left(1-\beta_{n}\right) x_{n}+\beta_{n} S x_{n}-S x_{n}\right\| \\
\leq & (1+c)\left\|x_{n}-T y_{n}\right\|+a \beta_{n}\left\|x_{n}-S x_{n}\right\| \\
& +b\left\|x_{n}-S x_{n}\right\|+b \beta_{n}\left\|x_{n}-S x_{n}\right\|+b\left\|x_{n}-T y_{n}\right\| \\
& +c\left(1-\beta_{n}\right)\left\|x_{n}-S x_{n}\right\| \\
= & (1+b+c)\left\|x_{n}-T y_{n}\right\| \\
& +\left(a \beta_{n}+b+b \beta_{n}+c\left(1-\beta_{n}\right)\right)\left\|x_{n}-S x_{n}\right\|
\end{aligned}
$$

we have

$(2.2)\left(1-a \beta_{n}-b-b \beta_{n}-c\left(1-\beta_{n}\right)\right)\left\|x_{n}-S x_{n}\right\| \leq(1+b+c)\left\|x_{n}-T y_{n}\right\|$

Let $M_{1}=1-a \beta_{n}-b-b \beta_{n}-c\left(1-\beta_{n}\right)$, then

$$
\begin{aligned}
M_{1}= & 1-a \beta_{n}-b-b \beta_{n}-c+c \beta_{n} \\
= & 1-b-c-(a+b-c) \beta_{n} \\
\geq & a+b+c-(a+b-c) \beta_{n} \\
> & 0
\end{aligned}
$$

so

$$
\left\|x_{n}-S x_{n}\right\| \leq \frac{1+b+c}{M_{1}}\left\|x_{n}-T y_{n}\right\|
$$


if $\lim _{n \rightarrow \infty}$ inf $\left\|x_{n}-T y_{n}\right\|=0$, there exists subsequence $\left\|x_{n_{k}}-T y_{n_{k}}\right\| \rightarrow 0$ when $n_{k} \rightarrow \infty$, by $(2.3)$ we have $\lim _{n_{k} \rightarrow \infty}\left\|x_{n_{k}}-S x_{n_{k}}\right\|=0$.

Next we will show that sequence $\left\{S x_{n_{k}}\right\}$ is a Cauchy sequence. For any $n_{k_{1}}, n_{k_{2}}$, we have

$$
\begin{aligned}
& \left\|S x_{n_{k_{1}}}-S x_{n_{k_{2}}}\right\| \\
\leq \quad & \left\|S x_{n_{k_{1}}}-T y_{n_{k_{2}}}\right\|+\left\|S x_{n_{k_{2}}}-T y_{n_{k_{2}}}\right\| \\
\leq \quad & a\left\|x_{n_{k_{1}}}-y_{n_{k_{2}}}\right\|+b\left\{\left\|x_{n_{k_{1}}}-S x_{n_{k_{1}}}\right\|+\left\|y_{n_{k_{2}}}-T y_{n_{k_{2}}}\right\|\right\} \\
& +c\left\{\left\|x_{n_{k_{1}}}-T y_{n_{k_{2}}}\right\|+\left\|y_{n_{k_{2}}}-S x_{n_{k_{1}}}\right\|\right\} \\
& +\left\|S x_{n_{k_{2}}}-T y_{n_{k_{2}}}\right\| \\
\leq \quad & a\left\{\left\|x_{n_{k_{1}}}-S x_{n_{k_{1}}}\right\|+\left\|S x_{n_{k_{1}}}-S x_{n_{k_{2}}}\right\|+\left\|S x_{n_{k_{2}}}-y_{n_{k_{2}}}\right\|\right\} \\
& +b\left\{\left\|x_{n_{k_{1}}}-S x_{n_{k_{1}}}\right\|+\left\|y_{n_{k_{2}}}-T y_{n_{k_{2}}}\right\|\right\} \\
& +c\left\{\left\|x_{n_{k_{1}}}-S x_{n_{k_{1}}}\right\|+\left\|S x_{n_{k_{1}}}-S x_{n_{k_{2}}}\right\|\right. \\
& +\left\|S x_{n_{k_{2}}}-T y_{n_{k_{2}}}\right\|+\left\|y_{n_{k_{2}}}-S x_{n_{k_{2}}}\right\| \\
& \left.+\left\|S x_{n_{k_{2}}}-S x_{n_{k_{1}}}\right\|\right\}+\left\|S x_{n_{k_{2}}}-T y_{n_{k_{2}}}\right\|
\end{aligned}
$$

Since $b>0$, thus obviously we have $1-a-2 c>0$. Simplify, then we get

$$
\begin{aligned}
\left\|S x_{n_{k_{1}}}-S x_{n_{k_{2}}}\right\| \leq & R_{1}\left\|x_{n_{k_{1}}}-S x_{n_{k_{1}}}\right\|+R_{2}\left\|y_{n_{k_{2}}}-T y_{n_{k_{2}}}\right\| \\
& +R_{3}\left\|y_{n_{k_{2}}}-S x_{n_{k_{2}}}\right\|+R_{4}\left\|S x_{n_{k_{2}}}-T y_{n_{k_{2}}}\right\|
\end{aligned}
$$

Where $R_{1}=\frac{a+b+c}{1-a-2 c} \geq 0, R_{2}=\frac{b}{1-a-2 c} \geq 0, R_{3}=\frac{a+c}{1-a-2 c} \geq 0$, and $R_{4}=\frac{1+c}{1-a-2 c} \geq 0$

According to Ishikawa iteration

$$
\begin{aligned}
y_{n} & =\left(1-\beta_{n}\right) x_{n}+\beta_{n} S x_{n} \\
x_{n+1} & =\left(1-\alpha_{n}\right) x_{n}+\alpha_{n} T y_{n}
\end{aligned}
$$

It is easy to see that $\left\|x_{n_{k}}-y_{n_{k}}\right\|=\beta_{n_{k}}\left\|x_{n_{k}}-S x_{n_{k}}\right\|$ and $\left\|S x_{n_{k}}-y_{n_{k}}\right\|$ $=\left(1-\beta_{n_{k}}\right)\left\|x_{n_{k}}-S x_{n_{k}}\right\|$. Then we consider the sequence $\left\|T y_{n_{k}}-S x_{n_{k}}\right\|$,

$$
\begin{aligned}
\left\|y_{n_{k}}-T y_{n_{k}}\right\| & =\left\|\left(1-\beta_{n_{k}}\right) x_{n_{k}}+\beta_{n} S x_{n_{k}}-T y_{n_{k}}\right\| \\
& \leq\left(1-\beta_{n_{k}}\right)\left\|x_{n_{k}}-T y_{n_{k}}\right\|+\beta_{n_{k}}\left\|S x_{n_{k}}-T y_{n_{k}}\right\|
\end{aligned}
$$


From the definition of the mean nonexpansive mappings, we obtain

$$
\begin{aligned}
\left\|T y_{n_{k}}-S x_{n_{k}}\right\| \leq & a\left\|x_{n_{k}}-y_{n_{k}}\right\|+b\left\{\left\|x_{n_{k}}-S x_{n_{k}}\right\|+\left\|y_{n_{k}}-T y_{n_{k}}\right\|\right\} \\
& +c\left\{\left\|x_{n_{k}}-T y_{n_{k}}\right\|+\left\|y_{n_{k}}-S x_{n_{k}}\right\|\right\} \\
\leq \quad & a \beta_{n_{k}}\left\|x_{n_{k}}-S x_{n_{k}}\right\|+b\left\|x_{n_{k}}-S x_{n_{k}}\right\| \\
& +b\left(1-\beta_{n_{k}}\right)\left\|x_{n_{k}}-T y_{n_{k}}\right\|+b \beta_{n_{k}}\left\|S x_{n_{k}}-T y_{n_{k}}\right\| \\
& +c\left\|x_{n_{k}}-T y_{n_{k}}\right\|+c\left(1-\beta_{n_{k}}\right)\left\|x_{n_{k}}-S x_{n_{k}}\right\| \\
= & \left(a \beta_{n_{k}}+b+c\left(1-\beta_{n_{k}}\right)\right)\left\|x_{n_{k}}-S x_{n_{k}}\right\| \\
& +\left(b\left(1-\beta_{n_{k}}\right)+c\right)\left\|x_{n_{k}}-T y_{n_{k}}\right\|+b \beta_{n_{k}}\left\|S x_{n_{k}}-T y_{n_{k}}\right\|
\end{aligned}
$$

Since

$$
\left\|x_{n_{k}}-S x_{n_{k}}\right\| \leq\left\|S x_{n_{k}}-T y_{n_{k}}\right\|+\left\|x_{n_{k}}-T y_{n_{k}}\right\|
$$

we can get the following inequality

$$
\begin{aligned}
\left\|T y_{n_{k}}-S x_{n_{k}}\right\| \leq & \left(b\left(1-\beta_{n_{k}}\right)+c+a \beta_{n_{k}}+b+c\left(1-\beta_{n_{k}}\right)\right)\left\|x_{n_{k}}-T y_{n_{k}}\right\| \\
& +\left(b \beta_{n_{k}}+a \beta_{n_{k}}+b+c\left(1-\beta_{n_{k}}\right)\right)\left\|S x_{n_{k}}-T y_{n_{k}}\right\|
\end{aligned}
$$

Therefor

$$
\begin{aligned}
& \left(1-b-c-(a+b-c) \beta_{n_{k}}\right)\left\|T y_{n_{k}}-S x_{n_{k}}\right\| \\
& \leq\left(b\left(1-\beta_{n_{k}}\right)+c+a \beta_{n_{k}}+b+c\left(1-\beta_{n_{k}}\right)\right)\left\|x_{n_{k}}-T y_{n_{k}}\right\|
\end{aligned}
$$

Since $0 \leq \beta_{n_{k}} \leq 1$, so we can get $1-b-c-(a+b-c) \beta_{n_{k}} \geq(a+b)\left(1-\beta_{n_{k}}\right)+c\left(1+\beta_{n_{k}}\right)>$ 0 , and it is easy to see that

$$
b\left(1-\beta_{n_{k}}\right)+c+a \beta_{n_{k}}+b+c\left(1-\beta_{n_{k}}\right)>0
$$

According to the condition $\left\|x_{n_{k}}-T y_{n_{k}}\right\| \rightarrow 0$, thus we get

$$
\lim _{n_{k} \rightarrow \infty}\left\|S x_{n_{k}}-T y_{n_{k}}\right\|=0 \text { and } \lim _{n_{k} \rightarrow \infty}\left\|y_{n_{k}}-T y_{n_{k}}\right\|=0
$$

So $\lim _{n_{k} \rightarrow \infty}\left\|x_{n_{k}}-y_{n_{k}}\right\|=\lim _{n_{k} \rightarrow \infty} \beta_{n_{k}}\left\|S x_{n_{k}}-x_{n_{k}}\right\|=0$.

We know

$$
\left\|x_{n_{k}}-S x_{n_{k}}\right\| \rightarrow 0,\left\|y_{n_{k}}-T y_{n_{k}}\right\| \rightarrow 0,\left\|S x_{n_{k}}-T y_{n_{k}}\right\| \rightarrow 0
$$

It is easy to see that $\left\|y_{n_{k}}-S x_{n_{k}}\right\| \rightarrow 0$, in particular, $\left\|x_{n_{k_{1}}}-S x_{n_{k_{1}}}\right\| \rightarrow 0, \| y_{n_{k_{2}}}-$ $T y_{n_{k_{2}}}\|\rightarrow 0,\| y_{n_{k_{2}}}-S x_{n_{k_{2}}}\|\rightarrow 0\|, S x_{n_{k_{2}}}-T y_{n_{k_{2}}} \| \rightarrow 0$ thus $\left\|S x_{n_{k_{1}}}-S x_{n_{k_{2}}}\right\|^{2} \rightarrow$ 0 , it is also say that $\left\{S x_{n_{k}}\right\}$ is a Cauchy sequence. We may assume that $p=$ $\lim _{n_{k} \rightarrow \infty} S x_{n_{k}}$, so we have $\lim _{n_{k} \rightarrow \infty} x_{n_{k}}=p$ using (1.4),we can get $\lim _{n \rightarrow \infty} x_{n}=p$ By (1.1), we have

$$
\begin{aligned}
\left\|S x_{n_{k}}-T p\right\| \leq & a\left\|x_{n_{k}}-p\right\|+b\left\{\left\|x_{n_{k}}-S x_{n_{k}}\right\|+\|p-T p\|\right\} \\
& +c\left\{\left\|x_{n_{k}}-T p\right\|+\left\|p-S x_{n_{k}}\right\|\right\}
\end{aligned}
$$


Let $n_{k} \rightarrow \infty$, we get

$$
\|p-T p\| \leq(b+c)\|p-T p\|
$$

Since $b+c<1$, that means $\|p-T p\|=0$, that is $T p=p, p$ is a fixed point of $T$. Similarly, we can prove that $S p=p$. thus, $\left\{x_{n}\right\}$ converges to the common fixed point of $S$ and $T$.

For the second step, we will prove the necessity, if $\left\{x_{n}\right\}$ converges to the common fixed point of $S$ and $T$, we assume that $\lim _{n \rightarrow \infty} x_{n}=p$,

$$
\begin{aligned}
\left\|x_{n_{k}}-T y_{n_{k}}\right\| & \leq\left\|x_{n_{k}}-p\right\|+\left\|T y_{n_{k}}-p\right\| \\
& \leq\left\|x_{n_{k}}-p\right\|+\left\|y_{n_{k}}-p\right\| \\
& \leq\left\|x_{n_{k}}-p\right\|+\left\|\left(1-\beta_{n_{k}}\right) x_{n_{k}}+\beta_{n_{k}} S x_{n_{k}}-p\right\| \\
& \leq\left\|x_{n_{k}}-p\right\|+\left(1-\beta_{n_{k}}\right)\left\|x_{n_{k}}-p\right\|+\beta_{n_{k}}\left\|S x_{n_{k}}-p\right\| \\
& \leq\left(1+1-\beta_{n_{k}}+\beta_{n_{k}}\right)\left\|x_{n_{k}}-p\right\| \\
& =2\left\|x_{n_{k}}-p\right\|
\end{aligned}
$$

Since $\lim _{n \rightarrow \infty} x_{n}=p$, thus $\lim _{n \rightarrow \infty}\left\|x_{n_{k}}-T y_{n_{k}}\right\|=0$, that is $\lim _{n \rightarrow \infty}$ inf $\| x_{n}-$ $T y_{n} \|=0$.

Corollary 2.2. Let $X$ be a Banach space, $S: X \rightarrow X$ and $T: X \rightarrow X$ are pair of mean non-expansive with a nonempty common fixed points set, if $b>0, c>0$, then the necessary and sufficient conditions that the Ishikawa sequence $\left\{x_{n}\right\}$ converges to the common fixed point of $S$ and $T$ is:

$$
\lim _{n \rightarrow \infty}\left\|x_{n}-T y_{n}\right\|=0 .
$$

Proof. If $\lim _{n \rightarrow \infty}\left\|x_{n}-T y_{n}\right\|=0$, then $\lim _{n \rightarrow \infty}$ inf $\left\|x_{n}-T y_{n}\right\|=0$. By Theorem 2.1 , we get $\lim _{n \rightarrow \infty} x_{n}=p$.

For the converse, we assume that $\lim _{n \rightarrow \infty} x_{n}=p$, then

$$
\begin{aligned}
\left\|x_{n}-T y_{n}\right\| & \leq\left\|x_{n}-p\right\|+\left\|T y_{n}-p\right\| \\
& \leq\left\|x_{n}-p\right\|+\left\|y_{n}-p\right\| \\
& \leq\left\|x_{n}-p\right\|+\left\|\left(1-\beta_{n}\right) x_{n}+\beta_{n} S x_{n}-p\right\| \\
& \leq\left\|x_{n}-p\right\|+\left(1-\beta_{n}\right)\left\|x_{n}-p\right\|+\beta_{n}\left\|S x_{n}-p\right\| \\
& \leq\left(1+1-\beta_{n}+\beta_{n}\right)\left\|x_{n}-p\right\| \\
& =2\left\|x_{n}-p\right\|
\end{aligned}
$$

Since $\lim _{n \rightarrow \infty} x_{n}=p$, thus $\lim _{n \rightarrow \infty}\left\|x_{n}-T y_{n}\right\|=0$.

The following theorem is a strong convergence theorem for Ishikawa iteration in uniformly convex Banach spaces. 
Theorem 2.3. Let $X$ be a uniformly convex Banach space, $S: X \rightarrow X$ and $T: X \rightarrow X$ are pair of mean non-expansive with a nonempty common fixed points set, if $b>0, c>0,0<\alpha \leq \alpha_{n} \leq \alpha^{\prime}<1$, then the Ishikawa sequence $\left\{x_{n}\right\}$ converges to the common fixed point of $S$ and $T$.

Proof. Let $p$ is a common fixed point of $S$ and $T$, by Lemma 1.3, we have

$$
\begin{aligned}
\left\|x_{n+1}-p\right\|^{2}= & \left\|\left(1-\alpha_{n}\right) x_{n}+\alpha_{n} T y_{n}-p\right\|^{2} \\
\leq & \alpha_{n}\left\|T y_{n}-p\right\|^{2}+\left(1-\alpha_{n}\right)\left\|x_{n}-p\right\|^{2} \\
& -\alpha_{n}\left(1-\alpha_{n}\right) \varphi\left(\left\|T y_{n}-x_{n}\right\|\right)
\end{aligned}
$$

However

$$
\begin{aligned}
\left\|y_{n}-p\right\| & =\left\|\left(1-\beta_{n}\right) x_{n}+\beta_{n} S x_{n}-p\right\| \\
& \leq\left(1-\beta_{n}\right)\left\|x_{n}-p\right\|+\beta_{n}\left\|S x_{n}-p\right\| \\
& \leq\left(1-\beta_{n}+\beta_{n}\right)\left\|x_{n}-p\right\| \\
& =\left\|x_{n}-p\right\|
\end{aligned}
$$

Therefore

$$
\left\|x_{n+1}-p\right\|^{2} \leq\left\|x_{n}-p\right\|^{2}-\alpha_{n}\left(1-\alpha_{n}\right) \varphi\left(\left\|T y_{n}-x_{n}\right\|\right)
$$

We can get

$$
\alpha_{n}\left(1-\alpha_{n}\right) \varphi\left(\left\|T y_{n}-x_{n}\right\|\right) \leq\left\|x_{n}-p\right\|^{2}-\left\|x_{n+1}-p\right\|^{2}
$$

Since $0<\alpha \leq \alpha_{n} \leq \alpha^{\prime}<1$, thus $\alpha_{n}\left(1-\alpha_{n}\right)>\alpha\left(1-\alpha^{\prime}\right)>0$. By Lemma 1.4, $\left\|x_{n}-p\right\|^{2}$ is a real decreasing bounded sequence, so $\left\|x_{n}-p\right\|^{2}$ converges.

Hence for any $\varepsilon>0$, there exists $n_{0} \in N$, such that whenever $n>n_{0}$, we have

$$
\alpha_{n}\left(1-\alpha_{n}\right) \varphi\left(\left\|T y_{n}-x_{n}\right\|\right) \leq\left\|x_{n}-p\right\|^{2}-\left\|x_{n+1}-p\right\|^{2}<\varepsilon
$$

thus $\varphi\left(\left\|T y_{n}-x_{n}\right\|\right) \rightarrow 0$, as $n \rightarrow \infty$, and hence $\left\|T y_{n}-x_{n}\right\| \rightarrow 0$, by the continuity and strictly increasing nature of $\varphi$. By Theorem 2.1, we get that $\left\{x_{n}\right\}$ converges to the common fixed point of $S$ and $T$, so that the conclusion of the theorem follows.

Between the pair of mappings, if $S=T$, then

$\|T x-T y\| \leq a\|x-y\|+b\{\|x-T x\|+\|y-T y\|\}+c\{\|x-T y\|+\|y-T x\|\}$ the mapping $\mathrm{T}$ is called mean nonexpansive mapping, we obtain the following.

Corollary 2.4. Let $X$ be a Banach space, $T: X \rightarrow X$ is a mean non-expansive with a nonempty fixed points set, if $b>0, c>0,0<\alpha \leq \alpha_{n} \leq \alpha^{\prime}<1$, then the Ishikawa sequence $\left\{x_{n}\right\}$ converges to the fixed point of $T$. 


\section{References}

[1] V. Berinde, On the convergence of the Ishikawa iteration in the class of quasi contractive operators, Acta Math. Univ. Comenian., 73(2004), 119-126.

[2] S. C. Bose, Common fixed points of mappings in a uniformly convex Banach space, J. London Math. Soc., 18(1978), 151-156.

[3] S. S. Chang, The fixed point theorem of mean non-expansive mapping in Banach space, (Chinese) Acta Sichuan Univsity, 2(1975), 67-78.

[4] Chen Jianren, Li Zhongkai, Banach operator pair and common fixed points for nonexpansive maps, Nonlinear Anal., 74(2011), 3086-3090.

[5] C. E. Chidume, Chika Moore, Fixed point iteration for pseudo-contractive maps, Proc. Amer. Math. Soc., 127(1999), 1163-1170.

[6] Lj. B. Ćirić, J. S. Ume and M. S. Khan, On the convergence of the Ishikawa iterates to a common fixed point of two mappings, Arch. Math., 39(2003), 123-127.

[7] R. Espínola, N. Hussain, Common fixed points for multimaps in metric spaces, Fixed Point Theory Appl, 2010(2010), Article ID 204981, 14 pages.

[8] S. Ishikawa, Fixed points by a new iterations method, Proc. Amer. Math. Soc., 44(1974), 147-150.

[9] Liu Qi Hou, A convergence theorem for Ishikawa iterates of continuous generalized nonexpansive maps, J. Math. Anal. Appl., 165(1992), 305-309.

[10] P-E. Maingé, Approximation methods for common fixed points of nonexpansive mappings in Hilbert spaces, J. Math. Anal. Appl., 325(2007), 469-479.

[11] R. A. Rashwan, On the convergence of Mann iterates to a common fixed point for a pair of mappings, Demonstratio. Math., 33(1990), 709-712.

[12] Song Yisheng, Chen Rudong, Iterative approximation to common fixed points of nonexpansive mapping sequences in reflexive Banach spaces, Nonlinear Anal., 66(2007), 591-603.

[13] Xu, Hong Kun, Inequalities in Banach spaces with applications, Nonlinear Anal, 16(1991), 1127-1138

[14] Zhaohui Gu, Yongjin Li, Approximation methods for common fixed points of mean nonexpansive mapping in Banach spaces, Fixed Point Theory and Applications, 2008 (2008), Article ID 471532, 7 pages. 